\title{
Análisis observacional del desarrollo del pensamiento computacional en Educación Infantil-3 años mediante una propuesta de resolución de problemas con un robot de suelo de direccionalidad programada
}

\section{Observational analysis of the development of computational thinking in Early Childhood Education -3 years old- through a proposal for solving problems with a ground robot with programmed directionality}

\author{
Marta Terroba \\ Universidad de La Rioja. Logroño, España \\ materra@unirioja.es \\ Juan Miguel Ribera \\ Universidad de La Rioja. Logroño, España \\ juan-miguel.ribera@unirioja.es \\ Daniel Lapresa \\ Universidad de La Rioja. Logroño, España \\ daniel.lapresa@unirioja.es \\ M. Teresa Anguera \\ Universidad de Barcelona. Barcelona, España \\ tanguera@ub.edu
}

\begin{abstract}
Resumen
Se presenta una secuencia de problemas de dificultad creciente para desarrollar el pensamiento computacional en el primer nivel del segundo ciclo de Educación Infantil -3 años- mediante un robot de suelo de direccionalidad programada. En el seno de la metodología observacional se ha conseguido caracterizar la capacidad de pensamiento computacional de los escolares de Educación Infantil-3años, en los diferentes problemas que conforman la propuesta de intervención diseñada. La fiabilidad de los datos, en forma de concordancia inter-observadores, ha sido garantizada mediante el coeficiente Kappa. Un análisis de generalizabilidad avala el muestreo observacional realizado. El desarrollo de las capacidades de organización espacial y autoevaluación del alumno, así como la intervención de la maestra, se han relevado como aspectos claves en la resolución de problemas matemáticos por medio del pensamiento computacional en Educación Infantil-3años.

Palabras clave: pensamiento computacional, Educación Infantil-3 años, robótica educativa, metodología observacional.
\end{abstract}

\begin{abstract}
A sequence of problems of increasing difficulty is presented to develop computational thinking in the first level of the second cycle of Early Childhood Education -3 years- by means of a programmed directionality ground robot. With the use the observational methodology, it has been possible to characterize the computational thinking capacity of Early Childhood Education -3 years oldschoolchildren, in the different problems that make up the intervention proposal designed. The reliability of the data has been guaranteed, in the form of inter-observer agreement, by means of the Cohen's Kappa coefficient. A generalizability analysis supports the observational sampling carried out. The development of the capacities of spatial organization and self-evaluation of the student, as well as the intervention of the teacher, have been revealed as key aspects in the resolution of mathematical problems through computational thinking in Early Childhood Education -3 years old-
\end{abstract}

Keywords: computational thinking, Early Childhood Education-3 years, educational robotics, observational methodology. 


\section{Introducción}

La expresión pensamiento computacional que acuñó Wing (2006) implica la resolución de problemas, el diseño de sistemas, y la comprensión de la conducta humana, haciendo uso de los conceptos fundamentales de la informática, debiendo ser considerada una destreza primordial para el ser humano y resultando fundamental en la resolución de problemas matemáticos. Posteriormente Wing (2008) aclaró que el concepto de pensamiento computacional integra el conjunto de fases de pensamiento incluidas en la resolución de problemas, de manera que los procesos implicados puedan abordarse por herramientas procesadoras de la información. Aho (2012) matizó que el pensamiento computacional incluye todos los procesos implicados en la formulación de problemas y que, por tanto, las soluciones pueden ser representadas en forma de pasos y algoritmos. De esta forma, el pensamiento computacional permite definir, comprender y resolver problemas mediante el uso de conceptos propios de la informática (Bers et al., 2019).

En los últimos años, el pensamiento computacional se ha convertido en una cuestión clave de la innovación educativa, haciendo de las escuelas entornos de alfabetización tecnológica (Bers et al., 2019; Manches y Plowman, 2017; Zapata-Ros, 2015). En el segundo ciclo de la etapa de Educación Infantil -entre tres y seis años-, se está trabajando para integrar la tecnología en las aulas, convirtiendo la enseñanza de la robótica y de la programación en materias accesibles y satisfactorias para los escolares (Barron et al., 2011; Bers et al., 2014). Los escolares del segundo ciclo de Educación Infantil tienen capacidad suficiente para desarrollar sencillos proyectos de robótica (Cejka et al., 2006; Kazakoff et al., 2013), aprender nociones de programación y desarrollar competencias de pensamiento computacional (Bers, 2008). Resulta de interés conocer las posibilidades que ofrece la robótica y la programación entre escolares más jóvenes en términos de desarrollo cognitivo, motriz y de interacción social (Bers, 2008; Bers et al., 2013; Lee et al., 2013) y, en concreto, en la resolución de problemas matemáticos (Brosterman, 1997; Resnick et al., 1998).

La generación de Tangible User Interfaces (Strawhacker y Bers, 2015) aporta múltiples posibilidades para el desarrollo del pensamiento computacional en Educación Infantil (Bers, 2008; Roger y Porstmore 2004). Está creciendo considerablemente el empleo de estos robots educativos, que permiten introducir lenguajes de programación (Avello et al, 2020; Kazakoff et al., 2013; Sullivan y Bers, 2016), contribuyendo a favorecer en el alumnado procesos de comprensión matemáticos, destrezas en la resolución de problemas y el desarrollo de habilidades de secuenciación (Bers, 2008; Diago et al., 2018; Kazakoff et al., 2013), competencias que tradicionalmente han sido trabajadas en las escuelas infantiles (Kazakoff y Bers, 2011). La integración de robots de suelo de direccionalidad programada se ve facilitada por su carácter intuitivo, que permite a los escolares de Educación Infantil -tres a seis añoscaracterizados por un incipiente pensamiento computacional, identificar rutinas, codificar, y reconocer errores de programación cuando el robot no realiza el camino diseñado. De este modo, los estudiantes construyen aprendizajes significativos que les ayudan a resolver situaciones problemáticas reales (Clements y Sarama, 1997; Fessakis et al., 2013; JiménezGestal et al., 2019) por medio de nociones computacionales (Wing, 2006) en un entorno lúdico propicio para avivar la indagación y potenciar el aprendizaje (Mazas et al., 2018).

En el presente trabajo se presenta una propuesta de intervención para el alumnado del primer nivel del segundo ciclo de Educación Infantil -3 años-, constituida por una secuencia de problemas de dificultad creciente a resolver mediante un robot de suelo de direccionalidad programada. El objetivo de este trabajo consiste en caracterizar el pensamiento computacional en el alumnado del primer curso de Educación Infantil -3 años-, durante la resolución de cada uno de los problemas de dificultad creciente que constituyen la propuesta de intervención.

Análisis observacional del desarrollo del pensamiento computacional en Educación Infantil-3 años mediante una propuesta de resolución de problemas con un robot de suelo de direccionalidad programada. Terroba, M., Ribera, J.M., Lapresa, D y Anguera, M.T.

Página $\mathbf{2}$ de $\mathbf{2 0}$ 


\section{Método}

El presente trabajo se ha desarrollado en el seno de la metodología observacional (Anguera, 1979). El diseño observacional es: nomotético -alumnos de Educación Infantil-3 años que actúan individualmente-; de seguimiento inter e intra-sesional -se registran las conductas objeto de estudio frame a frame a lo largo de la realización de los siete steps que constituyen la propuesta de resolución de problemas-; y multidimensional -lo que configura los diferentes criterios del instrumento de observación-. El grado de perceptividad es total (observación directa) en unas dimensiones, y parcial (observación indirecta) en otras (Anguera et al., 2018). La observación es participante, puesto que el primer autor del trabajo interactúa con los participantes.

\section{Participantes}

En el presente trabajo se ha realizado un muestreo intencional. Los participantes han sido 25 alumnos/as de la clase de Educación Infantil-3años del CEIP La Guindalera (Logroño, España). La edad de los 12 participantes que superaron la prueba de selección y han realizado la propuesta de intervención se corresponde con una media de 3,64 años y una desviación típica de 0,19 . El presente trabajo cuenta con los consentimientos informados pertinentes y con la aprobación del Comité de Ética de la Investigación de la Universidad de La Rioja (expediente CE-08-2020).

\section{Instrumento de observación}

El instrumento de observación ha sido elaborado ad hoc (Terroba et al, 2021). Es una combinación de formato de campo y sistemas de categorías (tabla 1), ya que en cada uno de los criterios del instrumento se han anidado sistemas de categorías que cumplen las condiciones de exhaustividad y mutua exclusividad (Anguera, 2003).

Tabla 1. Estructura resumida del instrumento de observación: criterios, categorías y códigos

\begin{tabular}{|c|c|}
\hline Criterios & Sistemas de categorías y códigos \\
\hline Step & $\begin{array}{l}\text { Step 1(TP1), step 2(TP2), step 3(TP3), step 4(TP4), step 5(TP5), step 6(TP6), step } \\
\text { 7(TP7) }\end{array}$ \\
\hline Fase & 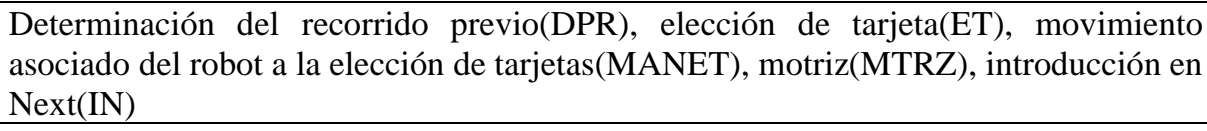 \\
\hline Intento intra-fase & Intento primero(I1), intento segundo(I2), intento tercero(I3) \\
\hline Eficacia intento/fase & $\begin{array}{l}\text { Resuelve(R), no resuelve(NR), concuerda con la fase de tarjetas pero no } \\
\text { resuelve(CFT), resuelve pero no concuerda con la determinación previa del } \\
\text { recorrido(RNDP) }\end{array}$ \\
\hline Paso & Primer paso(P1)...vigesimocuarto paso (P24) \\
\hline $\begin{array}{l}\text { Información espacial } \\
\text { del paso }\end{array}$ & Adelante(AD), atrás(AT), izquierda(GI), derecha(GD) \\
\hline $\begin{array}{l}\text { Orientación del robot } \\
\text { respecto a la posición } \\
\text { inicial }\end{array}$ & $\begin{array}{l}\text { Misma orientación(NPM), lateral izquierda(NPLI), lateral derecha(NPLD), en } \\
\text { espejo(NPE) }\end{array}$ \\
\hline $\begin{array}{l}\text { Orientación del robot } \\
\text { respecto al niño }\end{array}$ & $\begin{array}{l}\text { Misma orientación(NNM), lateral izquierda(NNLI), lateral derecha(NNLD), en } \\
\text { espejo(NNE) }\end{array}$ \\
\hline $\begin{array}{l}\text { Adaptación de la } \\
\text { conducta al problema } \\
\text { planteado }\end{array}$ & Adaptativa(ADAP), no adaptativa(NOAD) \\
\hline Tarjeta retirada & $\begin{array}{l}\text { Anterior(PA), hasta primer error(PPE), todas(PT), tarjeta incorrecta de la } \\
\text { secuencia(PTI), una tarjeta incorrecta de la secuencia(PUTI), una tarjeta correcta de la } \\
\text { secuencia(PUTC) }\end{array}$ \\
\hline $\begin{array}{l}\text { Instrucción de la } \\
\text { maestra }\end{array}$ & $\begin{array}{l}\text { Promueve el razonamiento(MRR), reconduce ante inacción(MRI), fija el error con una } \\
\text { pregunta(MFE), manifiesta explícitamente el error cometido pero no da la } \\
\text { respuesta(MENR), manifiesta explícitamente el error cometido y da la } \\
\text { respuesta(MEYR) }\end{array}$ \\
\hline
\end{tabular}

Análisis observacional del desarrollo del pensamiento computacional en Educación Infantil-3 años mediante una propuesta de resolución de problemas con un robot de suelo de direccionalidad programada. Terroba, M., Ribera, J.M., Lapresa, D y Anguera, M.T. 


\section{Procedimiento}

En primer lugar, se desarrolló una prueba de selección constituida por tres tareas básicas a realizar de forma motriz, cuya resolución correcta permitiera garantizar que los participantes tuvieran una capacidad de organización espacial y de resolución de problemas suficiente para afrontar la propuesta de intervención al completo: 1) problema de recorrido en línea recta; 2) recorrido con pocos pasos y con un giro; 3) recorrido con mayor número de pasos y con un giro. Las habilidades de secuenciación de pasos mostradas en las tareas de la prueba de selección no requieren de la abstracción necesaria en la resolución de problemas posteriores. Del total de los 25 participantes, 12 resolvieron de forma correcta las tres tareas propuestas y accedieron a la propuesta de intervención, formada por siete problemas, diseñados a modo de steps de dificultad creciente (véase Tabla 2). En el primero de ellos es el niño el que realiza el recorrido de forma motriz en una cuadrícula de tamaño apropiado a sus posibilidades de desplazamiento. En el resto de los steps el problema se resuelve mediante el robot de suelo de direccionalidad programada, requiriendo unos niveles superiores de abstracción (Wing, 2006). La secuencia avanza en dificultad desde los problemas de un único giro (problema 1 y 2) y de doble giro sobre camino marcado (problema 3), llegando a problemas de doble giro sin camino marcado (problemas 4 y 5), para terminar con problemas de solución abierta (problema 6, recorrido corto; y problema 7 de recorrido largo). La secuenciación de los problemas elegida permite a los aprendices incorporar los procedimientos de resolución exitosos y usarlos en planteamientos de mayor dificultad, mejorando sus habilidades de resolución de problemas.

Tabla 2. Estructura de la secuencia de problemas.

\begin{tabular}{|c|c|c|}
\hline Step & Problema & $\begin{array}{c}\text { Representación } \\
\text { gráfica }\end{array}$ \\
\hline Step 1 & $\begin{array}{l}\text { Recorrido a realizar de forma motriz, realizando los pasos de forma análoga } \\
\text { al robot, para llegar hasta el protagonista del contexto simbólico. La fase } \\
\text { motriz se realiza en una cuadrícula de tamaño apropiado a las posibilidades } \\
\text { de desplazamiento del niño }\end{array}$ & \begin{tabular}{|l|l|}
+45 \\
$\square \square$ \\
$\square \square$
\end{tabular} \\
\hline Step 2 & $\begin{array}{l}\text { Problema a resolver mediante el robot de suelo de direccionalidad } \\
\text { programada. El robot debe recorrer el camino señalado para llegar hasta el } \\
\text { protagonista del juego simbólico }\end{array}$ & 舟 \\
\hline Step 3 & $\begin{array}{l}\text { Problema a resolver mediante el robot de suelo de direccionalidad } \\
\text { programada. Problema que incluye con dos giros, para llegar hasta el } \\
\text { protagonista del juego simbólico }\end{array}$ & $\begin{array}{l}8 \\
\square \\
4\end{array}$ \\
\hline Step 4 & $\begin{array}{l}\text { Problema a resolver mediante el robot de suelo de direccionalidad } \\
\text { programada. No se presenta el camino marcado. Hay unas condiciones que } \\
\text { debe cumplir en su recorrido: recoger por orden al protagonista pequeño, al } \\
\text { mediano y al grande. Está prohibido pasar por las casillas en las que hay un } \\
\text { peligro }\end{array}$ &  \\
\hline Step 5 & $\begin{array}{l}\text { Problema a resolver mediante el robot de suelo de direccionalidad } \\
\text { programada. No se presenta el camino marcado. Hay unas condiciones que } \\
\text { debe cumplir en su recorrido: recoger por orden al protagonista pequeño, al } \\
\text { mediano y al grande }\end{array}$ & 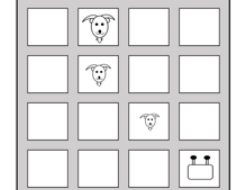 \\
\hline
\end{tabular}

Análisis observacional del desarrollo del pensamiento computacional en Educación Infantil-3 años mediante una propuesta de resolución de problemas con un robot de suelo de direccionalidad programada. Terroba, M., Ribera, J.M., Lapresa, D y Anguera, M.T. 
Problema a resolver mediante el robot de suelo de direccionalidad programada. Problema en el que el robot tiene que recorrer el camino más corto para llegar hasta el protagonista del juego simbólico

Problema a resolver mediante el robot de suelo de direccionalidad Step 7 programada. Problema en el que el robot tiene que recorrer el camino más largo para llegar hasta el protagonista del juego simbólico

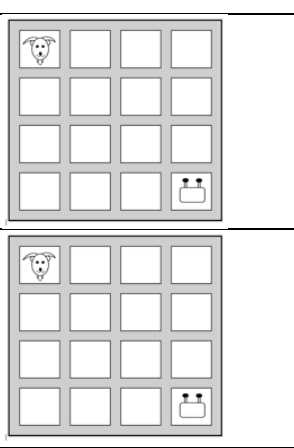

La intervención de la maestra juega un papel primordial a la hora de motivar e incentivar al alumnado en la búsqueda de soluciones de la secuencia de resolución de problemas planteada (Pöntinen y Räty-Záborszky, 2020; Sullivan y Bers, 2018). El protocolo de actuación de la maestra durante el desarrollo de la propuesta de intervención está enfocado a que sean los propios alumnos los que descubran los errores cometidos. El step se considera como no resuelto tras tres intentos fallidos, o al emplearse más de cinco minutos en su resolución. Los escolares pasan por todos los steps de la propuesta de intervención, aunque no se resuelvan correctamente.

\section{Registro y codificación}

Para el registro y la codificación de los 84 paquetes de datos que conforman el muestreo observacional -12 participantes resolviendo los siete steps de la propuesta de intervención-, se ha recurrido al software LINCE, versión 1.2.1 (Gabin et al., 2012) (véase figura 1).

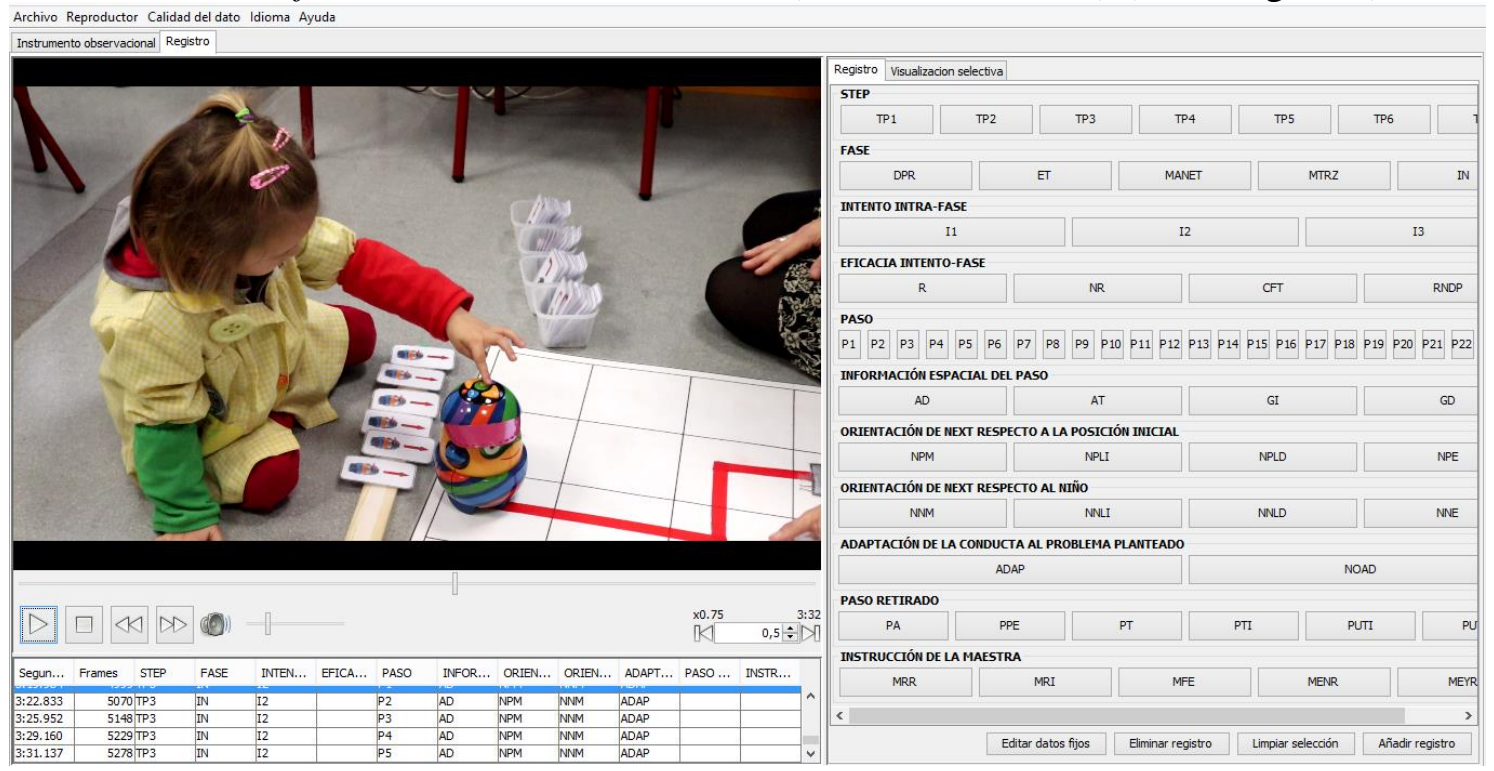

Figura 1. Captura de un momento del registro y codificación del paquete de datos correspondiente a la participante 5, step 3, en el software LINCE.

De acuerdo con Bakeman (1978) se han registrado datos concurrentes -tal y cómo se desprende del carácter multidimensional del diseño observacional, y de que el instrumento de observación sea una combinación de formato de campo y sistemas de categorías- y tiempobase (tipo IV). Asimismo, de acuerdo con Bakeman y Quera (1995) el tipo de datos es de multievento.

\section{Calidad del dato}

La constancia inter-sesional ha quedado garantizada a partir de la satisfacción, en cada una de las sesiones de observación, de la siguiente lista de mínimos: misma aula, materiales, y

Análisis observacional del desarrollo del pensamiento computacional en Educación Infantil-3 años mediante una propuesta de resolución de problemas con un robot de suelo de direccionalidad programada. Terroba, M., Ribera, J.M., Lapresa, D y Anguera, M.T. 
franja horaria de intervención; los participantes utilizan manguitos de colores -verde para la mano derecha y roja para la mano izquierda- para facilitar su organización espacial (véase figura 1).

\section{Fiabilidad de los datos}

Para determinar la fiabilidad, en forma de concordancia inter-observadores, se ha recurrido al coeficiente Kappa de Cohen (1960), mediante el programa informático LINCE. Dos han sido los observadores que han realizado el registro y codificación de los datos. Uno de ellos, el primer autor del presente trabajo que ha participado activamente en el desarrollo del sistema de observación. El segundo observador ha seguido un proceso de formación respetando las etapas propuestas por Anguera (2003). El primer observador registró la totalidad del muestreo observacional y el segundo un 16,66\% del muestreo observacional -la participación en la propuesta de intervención de dos participantes elegidos al azar-. Los valores del coeficiente Kappa de Cohen, se exponen a continuación -del step 1 al 7 de cada participante: participante $2(1,1,0,99,1,0,91,1$ y 1$)$; participante $12:(1,1,1,1,1,1$ y 0,90$)$.

Generalizabilidad de los resultados

La calidad del dato también se ha abordado en el seno de la teoría de la Generalizabilidad (Cronbach et al., 1972), mediante el software SAGT (Hernández-Mendo et al., 2016). Se han dispuesto de forma "cruzada" las facetas Categorías, con 65 niveles -las categorías correspondientes a los criterios variables del instrumento de observación-; Steps, con siete niveles; y Participantes, con 12 niveles. En las tres facetas se realiza la estimación para una población infinita. Se ha realizado el plan de medida [Categoría][Step]/[Participantes] para evaluar la generalizabilidad de los resultados a partir del número de participantes que han desarrollado la propuesta de intervención (tabla 3).

Tabla 3. Resultados del análisis de generalizabilidad correspondientes al plan de medida [Step][Categoría] /[Participante]

\begin{tabular}{lccccr}
\hline Fuentes de variación & Suma cuadrados & $\begin{array}{c}\text { Grados } \\
\text { libertad }\end{array}$ & $\begin{array}{c}\text { Cuadrado } \\
\text { medio }\end{array}$ & $\begin{array}{c}\text { \% } \\
\text { varianza }\end{array}$ & $\begin{array}{c}\text { Error } \\
\text { estándar }\end{array}$ \\
\hline [participante] & 1096,67 & 10 & 109,667 & 0,161 & 0,101 \\
[step] & 24645,157 & 6 & 4107,526 & 7,228 & 2,873 \\
[participante][step] & 3320,06 & 60 & 55,334 & 1,054 & 0,153 \\
[categorías] & 149481,431 & 64 & 2335,647 & 35,721 & 5,292 \\
[participante][categorías] & 5443,122 & 640 & 8,505 & 0,413 & 0,071 \\
[step][categorías] & 142334,479 & 384 & 370,663 & 46,396 & 2,426 \\
[participante][step][categorías] & 24742,875 & 3840 & 6,443 & 9,029 & 0,147 \\
\hline
\end{tabular}

El análisis de generalizabilidad muestra como la variabilidad queda asociada a la faceta Categorías (35,72\%), y a la faceta de interacción Step-Categorías (46,39\%). El análisis de los coeficientes de generalizabilidad determina que se consigue una fiabilidad de precisión de generalización (absoluta y relativa) de 0,989. Este resultado permite avalar la homogeneidad de la conducta desplegada por los participantes que constituyen el muestreo observacional.

\section{Análisis de los datos}

En el presente trabajo se han aplicado complementariamente dos técnicas de análisis diacrónico de datos, habituales en estudios observacionales, y que comparten como base de su cálculo los residuos ajustados: el análisis secuencial de retardos y de coordenadas polares.

El análisis secuencial de retardos permite contrastar la fuerza de la asociación entre categorías en registros en los que tiene lugar un seguimiento intrasesional (Anguera et al., in press). El análisis secuencial de retardos se ha realizado mediante el software GSEQ5 (Bakeman y Quera, 1995) que puede descargarse gratuitamente de https://www.mangold-

Análisis observacional del desarrollo del pensamiento computacional en Educación Infantil-3 años mediante una propuesta de resolución de problemas con un robot de suelo de direccionalidad programada. Terroba, M., Ribera, J.M., Lapresa, D y Anguera, M.T.

Página 6 de 20 
international.com/en/products/software/gseq. Este programa aplica una prueba binomial corregida mediante la propuesta de cálculo de la Z hipergeométrica de Allison y Liker (1982)que permite detectar las diferencias estadísticamente significativas existentes entre las probabilidades condicionadas -a partir de las frecuencias observadas- e incondicionadas -a partir de las frecuencias esperadas que indican el efecto del azar-, entre conductas given o criterio -la misma categoría que nos interesará como conducta focal en el análisis de coordenadas polares- y conductas target o condicionadas. Valores mayores que 1,96 $(p<0,05)$ representan una relación de activación entre la conducta given o criterio y la conducta target $\mathrm{o}$ condicionada, y, consecuentemente, las transiciones menores a -1,96 $(p<0,05)$ una relación de inhibición.

La técnica de análisis de coordenadas polares consigue reducir una importante cantidad de valores -los residuos ajustados correspondientes a la vertiente prospectiva y retrospectiva que la conducta focal tiene respecto a cada conducta condicionada- mediante el parámetro $Z_{\text {sum }}=\left(\sum \mathrm{Z} / \sqrt{\mathrm{n}}\right)$, introducido por Cochran (1954) y desarrollado por Sackett (1980). Deben contemplarse igual número de retardos prospectivos y retrospectivos; habitualmente de -5 a -1 y de +1 a +5 . Una vez calculados los parámetros $Z_{\text {sum }}$ prospectivos y retrospectivos correspondientes a cada díada formada por la conducta focal y cada conducta condicionada, se hallan:

a) La longitud del vector: es la distancia entre el origen de coordenadas $Z_{\text {sum }}(0,0)$ y el punto de intersección (en abscisas, el valor $Z_{\text {sum }}$ prospectivo; y en ordenadas, el valor $Z_{\text {sum }}$ retrospectivo); por tanto, la diagonal que configura la longitud del vector se obtiene calculando: $\sqrt{Z_{\text {sum }} \text { prospectivo }^{2}+Z_{\text {sum }} \text { retrospectivo }}{ }^{2}$. Se consideran significativas $(p<0,05)$ longitudes superiores a 1,96 .

b) El ángulo del vector: se corresponde con la función trigonométrica arco seno $\varphi=$ $\frac{Z_{\text {sum retrospectivo }}}{\text { longitud del vector }}$ En función del valor del arco seno $\varphi$, cada vector se incorpora en uno de los cuadrantes: cuadrante I $(0<\varphi<90)=\varphi$; cuadrante II $(90<\varphi<180)=180-\varphi$; cuadrante III $(180<\varphi<270)=180+\varphi$; cuadrante IV $(270<\varphi<360)=360-\varphi$.

Así, cada cuadrante refleja la activación/inhibición-prospectiva/retrospectiva entre la conducta focal y las conductas condicionadas (figura 2). El análisis de coordenadas polares se ve considerablemente facilitado por la implementación de un módulo específico en el software HOISAN (Hernández-Mendo et al., 2012), que puede descargarse de forma gratuita desde https://www.menpas.com.

Análisis observacional del desarrollo del pensamiento computacional en Educación Infantil-3 años mediante una propuesta de resolución de problemas con un robot de suelo de direccionalidad programada. Terroba, M., Ribera, J.M., Lapresa, D y Anguera, M.T. 




Figura 2: Representación gráfica de las relaciones de activación/inhibición-prospectiva/retrospectiva entre la conducta focal y conducta condicionada en función del cuadrante del mapa de coordenadas en el que se sitúa el vector.

\section{Resultados}

Dos han sido los análisis efectuados para satisfacer los objetivos del presente trabajo de investigación. Un análisis secuencial de retardos y un análisis de coordenadas polares (a partir de los resultados del análisis secuencial de retardos). El análisis secuencial de retardos se ha focalizado en el retardo 0 , y el análisis de coordenadas polares condensa la información correspondiente a los retardos $+1 \mathrm{a}+5 \mathrm{y}-1$ a -5 , pero no incorpora el análisis del retardo 0 .

El análisis del retardo 0, entre conductas de diferentes dimensiones, refleja la relación de activación o inhibición entre conductas given y targets correspondientes a eventos intra-fila del registro. Resulta especialmente relevante, en lo relativo al retardo 0, determinar las relaciones de activación e inhibición entre: a) las conductas correspondientes a la dimensión "fase" e "intento intra-fase" (que actúan como conductas given), y las conductas correspondientes a las dimensiones "eficacia intento/fase" y "paso retirado" (que actúan como conductas target) -véase tabla 4-; b) las conductas correspondientes a las dimensiones "información espacial del paso", "orientación del robot respecto a su posición inicial" y "orientación del robot respecto al niño" (conductas given), y las conductas constitutivas de la dimensión "adaptación de la conducta al problema planteado" (conductas target) -véase tabla $5-$.

Análisis observacional del desarrollo del pensamiento computacional en Educación Infantil-3 años mediante una propuesta de resolución de problemas con un robot de suelo de direccionalidad programada. Terroba, M., Ribera, J.M., Lapresa, D y Anguera, M.T. 
Tabla 4: Residuos ajustados estadísticamente significativos por step (TP) en el retardo 0 o coocurrencia, siendo las conductas given las categorías correspondientes a la dimensión "fase" e "intento intra-fase" (ver filas) y las conductas target las categorías de las dimensiones "eficacia intento/fase" y "paso retirado" (ver columnas).

\begin{tabular}{|c|c|c|c|c|c|c|c|c|}
\hline & $\mathbf{R}$ & NR & CFT & $\mathbf{P A}$ & PPE & PT & PTI & PUTC \\
\hline DPR & $\begin{array}{l}\text { TP5 }(2,43) \\
\text { TP6 }(3,1) \\
\text { TP7 }(5,36)\end{array}$ & TP7 $(-2,06)$ & & & & & & \\
\hline ET & ТР6(-2,01) & $\begin{array}{l}\text { TP2 }(3,09) \\
\text { TP3 }(3,18) \\
\text { TP5 }(3,28) \\
\text { TP6 }(4,09) \\
\text { TP7 }(3,07)\end{array}$ & & $\begin{array}{l}\text { TP1 }(-2,42) \\
\text { TP3(2) } \\
\text { TP6(2,24) }\end{array}$ & & TP3 $(-2,5)$ & TP5 $(2,42)$ & \\
\hline MANET & & $\begin{array}{l}\text { TP3 }(2,8) \\
\text { TP4 }(3,33) \\
\text { TP5 }(2,66) \\
\text { TP7(4) }\end{array}$ & & $\begin{array}{l}\text { TP4 }(3,25) \\
\text { TP5 }(2,42)\end{array}$ & ТР6(2,07) & $\begin{array}{l}\text { TP4 }(-3,25) \\
\text { TP5 }(-2,22) \\
\text { TP7 }(-3,2)\end{array}$ & & \\
\hline $\begin{array}{l}\text { MTRZ } \\
\text { IN }\end{array}$ & TP7 $(-2,5)$ & $\begin{array}{l}\text { TP2 }(-2,85) \\
\text { TP3 }(-5,15) \\
\text { TP4 }(-3,74) \\
\text { TP5 }(-4,23) \\
\text { TP6(-2,58) } \\
\text { TP7 }(-3,71)\end{array}$ & $\begin{array}{l}\text { TP1 }(2,09) \\
\text { TP2 }(3,31) \\
\text { TP3 }(5,17) \\
\text { TP4 }(4,1) \\
\text { TP5 }(4,95) \\
\text { TP6 }(4,3) \\
\text { TP7 }(6,45)\end{array}$ & $\mathrm{TP} 1(2,42)$ & & $\begin{array}{l}\text { TP2 }(2,58) \\
\text { TP3 }(3,62) \\
\text { TP4 }(3,25) \\
\text { TP5 }(3,03) \\
\text { TP6 }(2,58) \\
\text { TP7 }(3,69)\end{array}$ & & \\
\hline I1 & $\begin{array}{l}\text { TP3 }(-3,34) \\
\text { TP6 }(2,41)\end{array}$ & $\mathrm{TP} 1(2,18)$ & 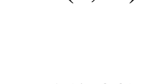 & $\begin{array}{l}\text { TP2 }(2,15) \\
\text { TP3 }(2,28)\end{array}$ & & $\begin{array}{l}\text { ТР5(-2,38) } \\
\text { TP7 }(-2,12)\end{array}$ & & TP5 $(2,55)$ \\
\hline $\mathrm{I} 2$ & $\begin{array}{l}\text { TP3 }(2,09) \\
\text { TP6 }(-2,4) \\
\text { TP7 }(-2,05)\end{array}$ & & TP1 $(2,03)$ & & & $\mathrm{TP} 7(2,12)$ & & \\
\hline I3 & $\begin{array}{l}\text { TP1 }(2,98) \\
\text { TP3 }(1,98) \\
\text { TP4 }(2,84)\end{array}$ & $\mathrm{TP} 1(-2,5)$ & & & & $\operatorname{TP5}(2,1)$ & & \\
\hline
\end{tabular}

Tabla 5. Residuos ajustados estadísticamente significativos por step (TP) en el retardo 0 o coocurrencia, siendo las conductas given las correspondientes a las dimensiones "información espacial del paso", "orientación del robot respecto a su posición inicial" y "orientación del robot respecto al niño"; y las conductas target las constitutivas de la dimensión "adaptación de la conducta al problema planteado".

\begin{tabular}{|c|c|c|}
\hline & Conducta adaptativa (ADAP) & Conducta no adaptativa (NOAD) \\
\hline $\mathrm{AD}$ & $\begin{array}{l}\text { TP1(2,42); TP2(4); TP3(2,74); ТP4(2,78); } \\
\text { TP5(2,03); ТР6(2,94) }\end{array}$ & $\begin{array}{l}\text { TP1(-2,42); TP2(-4); TP3(-2,74); TP4(-2,78); } \\
\text { TP5(-2,03); TP6(-2,94) }\end{array}$ \\
\hline $\mathrm{AT}$ & $\begin{array}{l}\text { TP2 }(-2,63) ; \text { ТР3 }(-3,7) ; \text { ТР4(-2,54); ТР5(-2,75); } \\
\text { ТP6(-2,87); ТР7(-3,17) }\end{array}$ & $\begin{array}{l}\text { ТР2(2,63); ТР3(3,7); ТР4(2,54); ТР5(2,75); } \\
\text { ТP6(2,87); ТP7(3,17) }\end{array}$ \\
\hline GI & ТP2 $(-3,42) ;$ ТP4 $(-2,83)$ & $\mathrm{TP} 2(3,42) ; \mathrm{TP} 4(2,83)$ \\
\hline GD & TP1 $(-2,91) ;$ ТP6(-2,58) & TP1 $(2,91) ;$ TP6(2,58) \\
\hline NPM & ТP2(3,58); ТP3(7,18); ТP4(5,78); ТP5(2,2) & ТP2(-3,58); ТР3(-7,18); ТР4(-5,78); ТР5(-2,2) \\
\hline NPLI & TP2 $(-2,52) ;$ TP3 $(-4,11) ;$ TP4 $(-5,14)$ & $\mathrm{TP} 2(2,52) ; \mathrm{TP} 3(4,11) ; \mathrm{TP} 4(5,14)$ \\
\hline NPLD & $\begin{array}{l}\text { TP2(-2,63); ТР3(-5,48); ТP4(-3,95); ТP5(-3,63); } \\
\text { ТP6(-4,5) }\end{array}$ & $\begin{array}{l}\text { ТP2 }(2,63) ; \mathrm{TP} 3(5,48) ; \mathrm{TP} 4(3,95) ; \mathrm{TP} 5(3,63) \\
\text { ТР6(4,5) }\end{array}$ \\
\hline NPE & $\begin{array}{l}\text { ТР1(-2,78); ТР2(-2,52); ТР3(-3,85); ТР5(-3,63); } \\
\text { ТР6(-5,52); ТР7(-3,69) }\end{array}$ & $\begin{array}{l}\text { ТР1 }(2,78) ; \text { ТР2 }(2,52) ; \mathrm{TP} 3(3,85) ; \mathrm{TP} 5(3,63) ; \\
\text { ТР6(5,52); ТР7(3,69) }\end{array}$ \\
\hline NNM & $\begin{array}{l}\text { TP1 }(2,94) ; \text { TP2 }(3,58) ; \text { TP3(7,84); ТP4(6,09); } \\
\text { TP5(3,08) }\end{array}$ & $\begin{array}{l}\text { TP1 }(-2,94) ; \text { TP2(-3,58); TP3(-7,84); ТP4(-6,09); } \\
\text { TP5(-3,08) }\end{array}$ \\
\hline NNLI & ТР1(-4,59); ТР2(-2,52); ТР3(-4,75); ТР4(-5,14) & ТP1(4,59); ТР2(2,52); ТР3(4,75); ТР4(5,14) \\
\hline NNLD & $\begin{array}{l}\text { ТP2(-2,63); ТР3(-5,48); ТP4(-4,24); ТP5(-2,25); } \\
\text { ТP6(-4,5) }\end{array}$ & $\begin{array}{l}\text { TP2 }(2,63) ; \text { TP3(5,48); ТP4(4,24); ТP5(2,25); } \\
\text { TP6(4,5) }\end{array}$ \\
\hline NNE & $\begin{array}{l}\text { TP1(-4,14); ТР2(-2,52); ТP3(-3,85); ТP5(-3,08); } \\
\text { TP6(-5,52); ТР7(-3,69) }\end{array}$ & $\begin{array}{l}\text { TP1 }(4,14) ; \text { TP2 }(2,52) ; \mathrm{TP} 3(3,85) ; \mathrm{TP} 5(3,08) \text {; } \\
\text { TP6(5,52); ТP7 }(-3,69)\end{array}$ \\
\hline
\end{tabular}

Análisis observacional del desarrollo del pensamiento computacional en Educación Infantil-3 años mediante una propuesta de resolución de problemas con un robot de suelo de direccionalidad programada. Terroba, M., Ribera, J.M., Lapresa, D y Anguera, M.T. 
Los resultados correspondientes al análisis de coordenadas polares permiten condensar la información de los retardos retrospectivos y prospectivos en un único vector, con lo que se facilita la presentación e interpretación de los resultados diacrónicos obtenidos (Lapresa et al., 2020). Resulta relevante el análisis de las coordenadas polares cuyos vectores son significativos en los que: a) como conducta focal se fijan las categorías correspondientes a las dimensiones "información espacial del paso", "orientación del robot respecto a su posición inicial" y "orientación del robot respecto al niño", y como conductas condicionadas las correspondientes a la dimensión "intervención de la maestra" -véase tabla 6-; b) como conductas focales se señalan las correspondientes a las dimensiones "información espacial del paso", "orientación del robot respecto a su posición inicial" y "orientación del robot respecto al niño", y como conductas condicionadas las correspondientes a la dimensión "paso retirado" -véase tabla 7-. 
RED. Revista de Educación a Distancia. Núm. 68, Vol. 21. Artíc. 10, 30-Noviembre -2021

DOI: http://dx.doi.org/10.6018/red.480411

Tabla 6: Parámetros correspondientes a los vectores significativos del análisis de coordenadas polares, tomando como conductas focales las correspondientes a las dimensiones "información espacial del paso", "orientación del robot respecto a su posición inicial" y "orientación del robot respecto al niño", y como conductas condicionadas las correspondientes a la dimensión "intervención de la maestra".

\begin{tabular}{|c|c|c|c|c|c|c|c|c|c|c|c|c|c|}
\hline Step & $\begin{array}{l}\text { Díada focal- } \\
\text { condicionada }\end{array}$ & Cuadrante & $\begin{array}{l}Z_{\text {sum }} \\
\text { prosp }\end{array}$ & $\begin{array}{l}Z_{\text {sum }} \\
\text { retro }\end{array}$ & Longitud & Ángulo & Step & $\begin{array}{l}\text { Díada focal- } \\
\text { condicionada }\end{array}$ & Cuadrante & $\begin{array}{l}Z_{\text {sum }} \\
\text { prosp }\end{array}$ & $\begin{array}{l}Z_{\text {sum }} \\
\text { retro }\end{array}$ & Longitud & Ángulo \\
\hline 1 & AD-MRR & IV & 1,95 & $-1,37$ & 2,39 & 324,91 & 6 & AD-MRR & IV & 2,3 & $-0,48$ & 2,35 & 348,13 \\
\hline 1 & AD-MENR & II & $-1,95$ & 1,37 & 2,39 & 144,91 & 6 & AD-MENR & IV & 0,57 & $-3,17$ & 3,22 & 280,16 \\
\hline 1 & GI-MRR & II & $-1,76$ & 1,37 & 2,23 & 142,07 & 6 & GI-MRR & II & $-2,86$ & 0,4 & 2,89 & 171,98 \\
\hline 1 & GI-MENR & IV & 1,76 & $-1,37$ & 2,23 & 322,07 & 6 & GI-MENR & IV & 2,86 & $-0,4$ & 2,89 & 351,98 \\
\hline 3 & NNLI-MRR & IV & 1,57 & $-3,13$ & 3,51 & 296,6 & 7 & AD-MRR & I & 2,45 & 0,94 & 2,62 & 20,97 \\
\hline 3 & NNLI-MFE & II & $-0,4$ & 2,67 & 2,69 & 98,49 & 7 & AD-MFE & II & $-2,73$ & 0 & 2,73 & 180 \\
\hline 3 & NNLI-MENR & II & $-1,36$ & 1,76 & 2,22 & 127,56 & 7 & AD-MENR & III & $-1,78$ & $-1,47$ & 2,31 & 219,51 \\
\hline 3 & NNM-MRR & II & $-2,21$ & 1,79 & 2,85 & 140,93 & 7 & GD-MRR & II & $-2,79$ & 1,37 & 3,11 & 153,8 \\
\hline 3 & NNM-MENR & IV & 1,9 & $-1,07$ & 2,18 & 330,55 & 7 & GD-MENR & I & 4,35 & 0,26 & 4,35 & 3,41 \\
\hline 3 & NPLI-MRR & IV & 1,57 & $-3,01$ & 3,39 & 297,58 & 7 & GI-MRR & III & $-1,06$ & $-2,91$ & 3,1 & 250 \\
\hline 3 & NPLI-MFE & II & $-0,4$ & 2,44 & 2,47 & 99,26 & 7 & GI-MFE & I & 3,5 & 1,04 & 3,65 & 16,59 \\
\hline 3 & NPLI-MENR & II & $-1,36$ & 1,78 & 2,23 & 127,35 & 7 & GI-MENR & II & $-1,17$ & 1,88 & 2,21 & 121,86 \\
\hline 3 & NPM-MRR & II & $-2,21$ & 1,81 & 2,86 & 140,65 & 7 & NNE-MRR & IV & 0,25 & $-2,88$ & 2,89 & 274,97 \\
\hline 3 & NPM-MENR & IV & 1,9 & $-1,17$ & 2,23 & 328,35 & 7 & NNE-MEYR & II & $-0,5$ & 2,55 & 2,6 & 101,02 \\
\hline 4 & GI-MFE & IV & 3,53 & $-0,4$ & 3,55 & 353,49 & 7 & NNLI-MFE & IV & 2,31 & $-0,15$ & 2,31 & 356,23 \\
\hline 4 & NNLD-MFE & III & $-1,34$ & $-1,77$ & 2,22 & 232,88 & 7 & NNLI-MENR & II & $-2,15$ & 0,26 & 2,16 & 173,11 \\
\hline 4 & $\begin{array}{l}\text { NNLD- } \\
\text { MENR }\end{array}$ & I & 0,89 & 2,03 & 2,22 & 66,38 & 7 & NNM-MFE & III & $-2,24$ & $-0,14$ & 2,24 & 183,54 \\
\hline 4 & NNLI-MFE & I & 0 & 2,44 & 2,44 & 90 & 7 & NNM-MENR & IV & 2,5 & $-0,08$ & 2,5 & 358,05 \\
\hline 4 & NPLD-MFE & III & $-1,27$ & $-2,11$ & 2,46 & 239 & 7 & NNM-MEYR & IV & 2,15 & $-0,25$ & 2,16 & 353,23 \\
\hline 4 & NPLD-MENR & I & 0,47 & 1,97 & 2,03 & 76,61 & 7 & NPE-MRR & IV & 0,25 & $-2,88$ & 2,89 & 274,97 \\
\hline 4 & NPLI-MFE & I & 0 & 2,44 & 2,44 & 90 & 7 & NPE-MEYR & II & $-0,5$ & 2,55 & 2,6 & 101,02 \\
\hline 5 & NNLI-MRR & IV & 0,51 & $-2,29$ & 2,34 & 282,45 & 7 & NPLI-MFE & IV & 2,31 & $-0,29$ & 2,33 & 352,93 \\
\hline 5 & NNLI-MENR & II & $-0,4$ & 2,34 & 2,38 & 99,64 & 7 & NPLI-MENR & II & $-2,15$ & 0,65 & 2,24 & 163,19 \\
\hline 5 & NNM-MENR & IV & 0,4 & $-1,93$ & 1,97 & 281,67 & 7 & NPLI-MEYR & III & $-1,76$ & $-0,89$ & 1,98 & 206,91 \\
\hline
\end{tabular}

Análisis observacional del desarrollo del pensamiento computacional en Educación Infantil-3 años mediante una propuesta de resolución de problemas con un robot de suelo de direccionalidad programada. Terroba, M., Ribera, J.M., Lapresa, D y Anguera, M.T. Página 11 de 20 
RED. Revista de Educación a Distancia. Núm. 68, Vol. 21. Artíc. 10, 30-Noviembre -2021

DOI: http://dx.doi.org/10.6018/red.480411

\begin{tabular}{lllllll|llllllll}
5 & NPLI-MRR & IV & 0,72 & $-3,02$ & 3,1 & 283,42 & 7 & NPM-MFE & III & $-2,24$ & $-0,03$ & 2,24 & 180,8 \\
5 & NPLI-MENR & II & $-0,57$ & 3,48 & 3,53 & 99,27 & 7 & NPM-MENR & IV & 2,5 & $-0,5$ & 2,54 & 348,75 \\
5 & NPM-MRR & II & $-0,72$ & 2,71 & 2,8 & 104,88 & 7 & NPM-MEYR & IV & 2,15 & $-0,19$ & 2,16 & 354,88 \\
5 & NPM-MENR & IV & 0,57 & $-3,17$ & 3,22 & 280,16 & & & & & & &
\end{tabular}

Tabla 7: Parámetros correspondientes a los vectores significativos del análisis de coordenadas polares, tomando como conductas focales las correspondientes a las dimensiones "información espacial del paso", "orientación del robot respecto a su posición inicial" y "orientación del robot respecto al niño", y como conductas condicionadas las correspondientes a la dimensión "paso retirado".

\begin{tabular}{|c|c|c|c|c|c|c|c|c|c|c|c|c|c|}
\hline Step & $\begin{array}{l}\text { Díada focal- } \\
\text { condicionada }\end{array}$ & Cuadrante & $\begin{array}{l}Z_{\text {sum }} \\
\text { prosp }\end{array}$ & $\begin{array}{l}\mathrm{Z}_{\text {sum }} \\
\text { retro }\end{array}$ & Longitud & Ángulo & Step & $\begin{array}{l}\text { Díada focal- } \\
\text { condicionada }\end{array}$ & Cuadrante & $\begin{array}{l}Z_{\text {sum }} \\
\text { prosp }\end{array}$ & $\begin{array}{l}\mathrm{Z}_{\text {sum }} \\
\text { retro }\end{array}$ & Longitud & Ángulo \\
\hline 1 & NNLD-PA & IV & 2,67 & $-0,59$ & 2,73 & 347,62 & 5 & NPM-PA & IV & 1,14 & $-3,01$ & 3,22 & 290,85 \\
\hline 1 & NNLD-PPE & II & $-1,44$ & 1,64 & 2,18 & 131,34 & 5 & NPM-PT & II & $-0,68$ & 3,67 & 3,73 & 100,56 \\
\hline 1 & NNM-PA & II & $-2,05$ & 0,12 & 2,06 & 176,63 & 6 & AD-PA & I & 2,46 & 1,07 & 2,69 & 23,54 \\
\hline 2 & GD-PA & II & $-1,97$ & 0,36 & 2,01 & 169,59 & 6 & AD-PPE & II & $-2,52$ & 0,76 & 2,63 & 163,29 \\
\hline 3 & GD-PA & II & $-2,69$ & 1,45 & 3,06 & 151,6 & 6 & GI-PA & III & $-2,71$ & $-0,69$ & 2,8 & 194,26 \\
\hline 3 & GD-PT & IV & 2,67 & $-2,1$ & 3,4 & 321,79 & 6 & GI-PPE & IV & 2,9 & $-0,64$ & 2,97 & 347,57 \\
\hline 3 & GI-PT & II & $-2,17$ & 0,17 & 2,17 & 175,58 & 6 & NNLI-PA & II & $-2,47$ & 1,54 & 2,91 & 147,99 \\
\hline 3 & NNLI-PA & I & 0,27 & 2,67 & 2,68 & 84,25 & 6 & NNLI-PT & IV & 2,8 & $-0,89$ & 2,93 & 342,42 \\
\hline 3 & NNLI-PPE & II & $-0,54$ & 2,84 & 2,89 & 100,72 & 6 & NNM-PA & IV & 3,14 & $-1,89$ & 3,66 & 328,99 \\
\hline 3 & NNLI-PT & IV & 0,44 & $-4,69$ & 4,71 & 275,34 & 6 & NNM-PT & II & $-3,56$ & 1,38 & 3,81 & 158,82 \\
\hline 3 & NNLI-PTI & II & $-0,78$ & 1,96 & 2,11 & 111,78 & 6 & NPLI-PA & II & $-2,47$ & 1,54 & 2,91 & 147,99 \\
\hline 3 & NNM-PA & IV & 0,6 & $-2,4$ & 2,47 & 284,06 & 6 & NPLI-PT & IV & 2,8 & $-0,89$ & 2,93 & 342,42 \\
\hline 3 & NNM-PT & II & $-1,42$ & 3,69 & 3,95 & 111,13 & 6 & NPM-PA & IV & 3,14 & $-1,54$ & 3,5 & 333,83 \\
\hline 3 & NPLI-PA & I & 0,56 & 3,22 & 3,27 & 80,07 & 6 & NPM-PT & II & $-3,56$ & 0,89 & 3,66 & 166,01 \\
\hline 3 & NPLI-PPE & II & $-0,57$ & 2,67 & 2,73 & 102,12 & 7 & AT-PUTI & IV & 2,38 & $-0,1$ & 2,39 & 357,53 \\
\hline 3 & NPLI-PT & IV & 0,18 & $-4,79$ & 4,79 & 272,19 & 7 & NNE-PPE & II & $-0,52$ & 4,12 & 4,16 & 97,17 \\
\hline 3 & NPM-PA & IV & 0,36 & $-3,43$ & 3,44 & 275,96 & 7 & NNE-PT & IV & 1,95 & $-2,13$ & 2,89 & 312,55 \\
\hline 3 & NPM-PT & II & $-1,3$ & 4,49 & 4,68 & 106,1 & 7 & NNLI-PA & I & 2,29 & 1,26 & 2,61 & 28,76 \\
\hline 4 & NNLD-PA & I & 0,86 & 4,38 & 4,46 & 78,9 & 7 & NNLI-PT & III & $-1,92$ & $-2,9$ & 3,48 & 236,53 \\
\hline 4 & NNLD-PT & III & $-0,86$ & $-4,38$ & 4,46 & 258,9 & 7 & NNLI-PUTC & I & 0,75 & 3,23 & 3,31 & 76,98 \\
\hline
\end{tabular}

Análisis observacional del desarrollo del pensamiento computacional en Educación Infantil-3 años mediante una propuesta de resolución de problemas con un robot de suelo de direccionalidad programada. Terroba, M., Ribera, J.M., Lapresa, D y Anguera, M.T. Página 12 de 20 
RED. Revista de Educación a Distancia. Núm. 68, Vol. 21. Artíc. 10, 30-Noviembre -2021 DOI: http://dx.doi.org/10.6018/red.480411

\begin{tabular}{|c|c|c|c|c|c|c|c|c|c|c|c|c|c|}
\hline 4 & NNM-PA & III & $-0,68$ & $-4,38$ & 4,43 & 261,12 & 7 & NNM-PA & III & $-1,74$ & $-1,52$ & 2,31 & 221,24 \\
\hline 4 & NNM-PT & I & 0,68 & 4,38 & 4,43 & 81,12 & 7 & NNM-PPE & IV & 1,51 & $-2,81$ & 3,19 & 298,29 \\
\hline 4 & NPLD-PA & I & 1,14 & 4,38 & 4,53 & 75,35 & 7 & NNM-PT & II & $-0,04$ & 3,72 & 3,72 & 90,62 \\
\hline 4 & NPLD-PT & III & $-1,14$ & $-4,38$ & 4,53 & 255,35 & 7 & NNM-PUTC & IV & 0,15 & $-2,46$ & 2,46 & 273,44 \\
\hline 4 & NPM-PA & III & $-0,97$ & $-4,38$ & 4,48 & 257,5 & 7 & NPE-PPE & II & $-0,52$ & 4,12 & 4,16 & 97,17 \\
\hline 4 & NPM-PT & I & 0,97 & 4,38 & 4,48 & 77,5 & 7 & NPE-PT & IV & 1,95 & $-2,13$ & 2,89 & 312,55 \\
\hline 5 & GD-PUTC & II & $-0,43$ & 2,18 & 2,23 & 101,24 & 7 & NPLI-PA & I & 2,29 & 1,76 & 2,89 & 37,51 \\
\hline 5 & NNLI-PA & II & $-0,99$ & 3,01 & 3,16 & 108,2 & 7 & NPLI-PT & III & $-1,92$ & $-3,13$ & 3,67 & 238,46 \\
\hline 5 & NNLI-PT & IV & 0,23 & $-3,04$ & 3,05 & 274,38 & 7 & NPLI-PUTC & I & 0,75 & 3,15 & 3,24 & 76,66 \\
\hline 5 & NNM-PA & IV & 1,14 & $-3,01$ & 3,22 & 290,85 & 7 & NPM-PA & III & $-1,74$ & -2 & 2,65 & 229,03 \\
\hline 5 & NNM-PT & II & $-0,68$ & 3,04 & 3,11 & 102,7 & 7 & NPM-PPE & IV & 1,51 & $-2,75$ & 3,13 & 298,83 \\
\hline 5 & NPLI-PA & II & $-0,99$ & 3,01 & 3,16 & 108,2 & 7 & NPM-PT & II & $-0,04$ & 3,95 & 3,95 & 90,58 \\
\hline 5 & NPLI-PT & IV & 0,23 & $-3,67$ & 3,68 & 273,62 & 7 & NPM-PUTC & IV & 0,15 & $-2,39$ & 2,4 & 273,53 \\
\hline
\end{tabular}




\section{Discusión}

En el trabajo presentado se ha desarrollado una propuesta de intervención para la resolución de problemas mediante el desarrollo del pensamiento computacional en Educación Infantil a través del uso de un robot de suelo de direccionalidad programada. Los resultados obtenidos han permitido identificar la capacidad de pensamiento computacional de los escolares de $1^{\circ}$ de Educación Infantil -3años-, en los diferentes problemas que conforman la propuesta de intervención diseñada. La homogeneidad de los datos que se desprende de los resultados del análisis de generalizabilidad realizado (Blanco-Villaseñor, 1993) permiten superar la limitación que supone el número de participantes y justifica el muestreo observacional efectuado.

El análisis secuencial de retardos en la coocurrencia de las conductas given correspondientes a las dimensiones "fase" e "intento intra-fase" y las conductas target correspondientes a las dimensiones "eficacia intento/fase" y "paso retirado" -véase tabla 4-, señala las dificultades con la que se encuentran los participantes en cada fase de la resolución de los problemas. La asociación con la conducta condicionada no resuelve (NR), refleja la dificultad que supone para Educación Infantil-3 años, las fases que conllevan un proceso de elección de tarjetas -“elección de tarjeta" (ET) y "movimiento asociado del robot a la elección de tarjetas" (MANET)- en todos los steps que deben ser resueltos mediante el robot (steps 2,3,4,5,6 y 7). La fase "introducción en el robot" (IN) muestra una asociación significativa con la conducta "concuerda con la fase de tarjetas pero no resuelve" (CFT), que pone de manifiesto la ausencia de dificultad a la hora de introducir los códigos de las tarjetas seleccionadas en el robot, superando sin problema el paso de la codificación a la programación del robot (Bers, 2018).

En la fase de "introducción en el robot" (IN), condicionada por la lógica interna del funcionamiento del robot, se produce una asociación con la conducta retirada de "todas las tarjetas" (PT), viéndose los escolares forzados, cuando se produce un error en la resolución, a borrar toda la secuencia de comandos introducidos en el robot. En la fase "motriz" (MTRZ), que sólo tiene lugar en el step 1, los participantes son capaces de descubrir el error al efectuar el recorrido motrizmente y retirar la tarjeta anterior cuando es errónea (PA). Sin embargo, en las fases que conllevan elección de tarjetas -"elección de tarjeta" (ET) y "movimiento asociado del robot a la elección de tarjetas" (MANET)se ha detectado una relación de inhibición con la retirada de todas las tarjetas (PT). Pese a la dificultad que supone esta fase, los escolares son capaces de identificar los errores cometidos y retirar la tarjeta anterior errónea (PA) -fase ET de los steps 3 y 6; y fase MANET de los steps 4 y 5-, la tarjeta incorrecta de la secuencia (PTI) -fase ET del step 5-, o la retirada de tarjetas hasta el primer error (PPE) -fase MANET del step 6-. Estos resultados revelan la competencia de los escolares de Educación Infantil-3 años en la autocorrección de los fallos cometidos en la codificación del problema (Diago et al., 2018), gracias a la posibilidad que ofrece el robot de autoevaluación de la solución generada.

En lo relativo al número de intentos intra-fase -tabla 4- se ha detectado una asociación significativa a que: el step 6 se resuelva en el primer intento (I1); el step 3, en el segundo intento (I2); mientras que en los steps 1,3 y 4 la asociación es con la resolución al tercer intento (I3). Estos resultados reflejan cómo los escolares incorporan el aprendizaje adquirido a lo largo de los steps previos, lo que les permite superar retos de mayor dificultad, a medida que se resuelven los problemas anteriores (Sinclair, 2005). En los dos primeros steps en los que la resolución del problema se lleva a cabo con el robot -steps 2 y 3-, los escolares son capaces de descubrir los errores de su pensamiento computacional en el primer intento (I1) sin necesidad de retirar todas las tarjetas y tener

Análisis observacional del desarrollo del pensamiento computacional en Educación Infantil-3 años mediante una propuesta de resolución de problemas con un robot de suelo de direccionalidad programada. Terroba, M., Ribera, J.M., Lapresa, D y Anguera, M.T. 
que empezar de nuevo, como sucede en steps de mayor complejidad -intento segundo del step 7 (I2) y tercero del step 5 (I3)-.

En la etapa de Educación Infantil se considera esencial facilitar la ejecución de actividades que posibiliten a los escolares la interacción con el espacio y les permita realizar una contextualización de los problemas, transformándolos en situaciones reales que provean de significatividad al aprendizaje (Belasko et al., 2019; Jiménez-Gestal et al., 2019). El análisis secuencial de retardos en la coocurrencia de las conductas given correspondientes a las dimensiones "información espacial del paso", "orientación del robot respecto a su posición inicial" y "orientación del robot respecto al niño" y las conductas target constitutivas de la dimensión "adaptación de la conducta al problema planteado" -véase tabla 5- muestra las dificultades que conlleva, para el pensamiento computacional del niño de 3 años, su incipiente capacidad de organización espacial (Denis, 2017). Los resultados obtenidos reflejan que la información espacial "adelante" (AD) no entraña mayor dificultad -asociación con respuesta adaptativa (ADAP) e inhibición respuesta no adaptativa en los steps 1 al 6-. Sin embargo, el desplazamiento "atrás" (AT) -asociación con respuesta no adaptativa (NOAD) e inhibición con respuesta adaptativa en los steps 2 al 6-, afecta seriamente la competencia en el pensamiento computacional de los participantes. La información espacial correspondiente a los giros también implica dificultades: el giro a la derecha (GD) -en los steps 1 y 6- y el giro a la izquierda (GI) -en los steps 2 y 4-, presentan asociación con una respuesta no adaptativa (NOAD) e inhibición con adaptativa (ADAP). Cuando el robot se encuentra en la misma orientación que la del niño (NNM) o en la posición inicial de salida (NPM) el niño no encuentra dificultades para dar una respuesta adaptativa al problema (ADAP). Sin embargo, la información espacial del paso atrás (AT), la orientación del robot respecto al niño lateral izquierda (NNLI), lateral derecha (NNLD) o en espejo (NNE), así como la posición del robot respecto a su posición de inicio lateral izquierda (NPLI), lateral derecha (NPLD) o en espejo (NPE) activan una respuesta no adaptativa (NOAD). Estos resultados reflejan las dificultades de organización espacial del alumnado de Educación Infantil-3 años, en la resolución de un problema que implique descentramiento al tener que utilizar diversas referencias espaciales al mismo tiempo (Denis, 2017).

Resulta especialmente relevante el papel de la maestra para generar ayudas al alumnado en la resolución de problemas (Giaconi et al., 2018; Pifarré y Sanuy, 2002), así como para incentivar y provocar desafíos que alienten su aprendizaje (Pöntinen y RätyZáborszky, 2020). Los resultados de los análisis de coordenadas polares -tabla 6-, muestran vectores significativos en los steps 1,3,4,5,6 y 7. Cuando la información espacial del paso es "adelante" (AD) la maestra interviene -en los steps 1, 6 y 7promoviendo el razonamiento (MRR). Sin embargo, cuando la información espacial del paso es giro a la izquierda (GI), la maestra manifiesta el error cometido sin dar la respuesta (MENR) -en los steps 1 y 6- o fija la atención del niño en el error cometido (MFE) por medio de preguntas -en el step 7-. Cuando el paso se corresponde con una misma orientación del robot respecto al niño (NNM) o igual a la posición inicial (NPM), se ha detectado -en los steps 3,5 y 7- una activación prospectiva a que la maestra manifieste el error cometido, pero no dé la respuesta (MENR). En el step 7 se ha detectado la activación prospectiva a que la maestra haga manifiesto el error y dé la respuesta correcta del paso al alumno (MEYR), lo que revela la dificultad de dicho step para los alumnos de Educación Infantil-3 años. Cuando la orientación del robot es lateral izquierda respecto al niño (NNLI) o respecto a su posición inicial (NPLI), en los steps 4 y 7 se produce una activación prospectiva a que la maestra manifieste explícitamente el error cometido pero no dé la respuesta (MFE). El alumnado de Educación Infantil-3 años precisa de la

Análisis observacional del desarrollo del pensamiento computacional en Educación Infantil-3 años mediante una propuesta de resolución de problemas con un robot de suelo de direccionalidad programada. Terroba, M., Ribera, J.M., Lapresa, D y Anguera, M.T.

Página 15 de 20 
intervención de la maestra para descubrir los errores cometidos en la secuenciación de los pasos de la solución problema y en la programación.

El análisis de coordenadas polares -tomando como conductas focales las correspondientes a las dimensiones "información espacial del paso", "orientación del robot respecto a su posición inicial" y "orientación del robot respecto al niño", y como conductas condicionadas las correspondientes a la dimensión "paso retirado" (véase tabla 7)- refleja cómo el uso de este tipo de robots permite a los escolares realizar una propuesta de programación por bloques y una evaluación del plan diseñado (Diago et al., 2018). En el step 1, la ejecución de la fase motriz permite a los participantes detectar el error cometido en la fase de elección de tarjetas y retirar la tarjeta anterior errónea (PA). Los steps que incorporan más de un giro en su resolución -steps 3,4,5,6 y 7- resultan más complicados para los alumnos de Educación Infantil-3 años, en función de su capacidad de descentramiento (Denis, 2017), y activan la retirada de todas las tarjetas (PT) cuando la orientación del robot respecto al niño (NNLI) o respecto a su posición inicial es lateral izquierda (NPLI) -steps 3, 5 y 6-; cuando la orientación del robot es la misma respecto a la posición inicial (NPM) o la misma respecto a la posición del niño (NNM)-en el step 4; o cuando el robot se encuentra en espejo respecto a la posición del niño (NNE) o a su posición inicial (NPE) -en el step 7-. Cuando el robot se encuentra orientado en la misma posición que el niño (NNM) o en la misma orientación respecto a su posición inicial (NPM), el niño es capaz de detectar el error o errores cometidos en la secuencia y retirar la tarjeta anterior (PA) -steps 3, 5 y 6-, o las tarjetas hasta el primer error (PPE) -step 7-. Los resultados señalan de nuevo la dificultad manifiesta que supone el último step de la propuesta diseñada para los niños de Educación Infantil-3 años; los participantes lejos de detectar los errores concretos cometidos, muestran una activación prospectiva a retirar tarjetas correctas de la secuencia (PUTC) cuando la orientación del robot es la misma que la del niño (NNM) o que su posición inicial (NPM), o cuando la orientación del robot es lateral izquierda (NNLI, NPLI).

En el presente trabajo se ha expuesto muestra una propuesta de intervención para el desarrollo del pensamiento computacional en Educación Infantil, mediante un robot de suelo con mandos de direccionalidad programada. En el seno de la metodología observacional se ha conseguido caracterizar el pensamiento computacional, en niños de primer curso de Educación Infantil-3 años, durante la resolución de cada uno de los problemas de dificultad creciente que constituyen la propuesta de intervención diseñada. El desarrollo de la capacidad de organización espacial de los escolares ha resultado una cuestión clave en la resolución de problemas matemáticos por medio del pensamiento computacional. La intervención de la maestra ha resultado fundamental para guiar al alumnado de Educación Infantil-3 años en el desarrollo de la propuesta de intervención (English y Watters, 2005; Lehrer et al., 2002). El movimiento efectuado por el robot a partir de la programación efectuada, ha permitido que el alumno realice una autoevaluación del plan diseñado (Bers, 2008; Diago et al., 2018).

El trabajo realizado arroja luz sobre el desarrollo del pensamiento computacional en el alumnado de Educación Infantil-3años a partir de una propuesta de intervención que permite a los escolares expresarse por medio de un lenguaje de programación (Sullivan y Bers, 2016). Las actividades presentadas son lúdicas, se han llevado a la práctica en un ambiente natural (Resnick et al., 1998) y resultan apropiadas para los escolares de Educación Infantil. Resultará muy interesante en futuros trabajos comprobar la evolución del pensamiento computacional del niño a lo largo de su progreso por los cursos superiores de Educación Infantil.

Análisis observacional del desarrollo del pensamiento computacional en Educación Infantil-3 años mediante una propuesta de resolución de problemas con un robot de suelo de direccionalidad programada. Terroba, M., Ribera, J.M., Lapresa, D y Anguera, M.T. 


\section{AGRADECIMIENTOS}

Los autores agradecen el apoyo del subproyecto Integration ways between qualitative and quantitative data, multiple case development, and synthesis review as main axis for an innovative future in physical activity and sports research [PGC2018-098742-B-C31] (2019-2021) (Ministerio de Ciencia, Innovación y Universidades / Agencia Estatal de Investigación / Fondo Europeo de Desarrollo Regional), que forma parte del proyecto coordinado New approach of research in physical activity and sport from mixed methods perspective (NARPAS_MM) [SPGC201800X098742CV0]. Asimismo, los autores agradecen el apoyo del grupo de investigación de la Generalitat de Catalunya, Grup de recerca i innovació en dissenys (GRID), Tecnología i aplicació multimedia i digital als dissenys observacionals [2017 SGR 1405]. Este estudio ha recibido también ayuda de fondos de investigación de la Universidad de La Rioja.

Presentación del artículo: 1 de julio 2021

Fecha de aprobación: 17 de noviembre de 2021

Fecha de publicación: 30 de noviembre de 2021

Terroba, M., Ribera, J.M., Lapresa, D y Anguera, M.T. (2021). Análisis observacional del desarrollo del pensamiento computacional en Educación Infantil-3 años mediante una propuesta de resolución de problemas con un robot de suelo de direccionalidad programada. RED. Revista de Educación a Distancia. Núm. 68, DOI: http://dx.doi.org/10.6018/red.490411

\section{Financiación}

Este trabajo no ha recibido ninguna subvención específica de los organismos de financiación en los sectores públicos, comerciales o sin fines de lucro

\section{Referencias}

Aho, A.V. (2012). Computation and computational thinking. The Computer Journal, 55(7), 832-835. https://doi.org/10.1093/comjn1/bxs074

Allison, P.D. y Liker, J.K. (1982). Analyzing sequential categorical data on dyadic interaction: A comment on Gottman. Psychological Bulletin, 93, 393-403. https://doi.org/10.1037/0033-2909.91.2.393

Anguera, M.T. (1979). Observational Typology. Quality \& Quantity. EuropeanAmerican Journal of Methodology, 13(6), 449-484. https://doi.org/10.1007/BF00222999

Anguera, M.T. (2003). La observación. En C. Moreno Rosset (Ed.), Evaluación psicológica. Concepto, proceso y aplicación en las áreas del desarrollo y de la inteligencia (pp. 271-308). Sanz y Torres.

Anguera, M.T., Portell, M., Chacón-Moscoso, S., y Sanduvete-Chaves, S. (2018). Indirect observation in everyday contexts: Concepts and methodological guidelines within a mixed methods framework. Frontiers in Psychology, 9:13. http://dx.doi.org/10.3389/fpsyg.2018.00013

Análisis observacional del desarrollo del pensamiento computacional en Educación Infantil-3 años mediante una propuesta de resolución de problemas con un robot de suelo de direccionalidad programada. Terroba, M., Ribera, J.M., Lapresa, D y Anguera, M.T. 
Anguera, M.T., Portell, P., Hernández-Mendo, A., Sánchez-Algarra, P., y Jonsson, G. K. (in press). Diachronic analysis of qualitative data. En A.J. Onwuegbuzie y B. Johnson (Eds.), Reviewer's Guide for Mixed Methods Research Analysis. Routledge.

Avello, R., Lavonen, J., y Zapata-Ros, M. (2020). Codificación y robótica educativa y su relación con el pensamiento computacional y creativo. Una revisión compresiva. RED. Revista de Educación a Distancia, 20(63). https://doi.org/10.6018/red.413021

Bakeman, R. (1978). Untangling streams of behavior: sequential analysis of observation data. En G.P. Sackett (Ed.) Observing Behaviour, Vol. II: Data Collection and Analysis Methods (pp. 63-78). University Park Press.

Bakeman, R. y Quera, V. (1995). Analyzing interaction: Sequential analysis with SDIS and GSEQ. Cambridge University Press.

Blanco-Villaseñor, A. (1993) Fiabilidad, precisión, validez y generalización de los diseños observacionales. En M.T. Anguera (Ed.) Metodología observacional en la investigación psicológica (pp. 149-261). P.P.U., Vol. II.

Barron, B., Cayton-Hodges, G., Bofferding, L., Copple, C., Darling-Hammond, L., y Levine, M. (2011). Take a Giant Step: A Blueprint for Teaching Children in a Digital Age. The Joan Ganz Cooney Center at Sesame Workshop.

Belasko, M., Herrán, E., \& Anguera, M.T. (2019). Dressing toddlers at the Emmi Pikler nursery school in Budapest: caregiver instrumental behavioral pattern. European Early Childhood Education Research Journal, 27(6), 972-887. https://doi.org/10.1080/1350293X.2019.1678928

Bers, M. (2008). Blocks, robots and computers: Learning about technology in early childhood. Teacher's College Press.

Bers, M. (2018). Coding as a Playground: Programming and Computational Thinking in the Early Childhood Classroom. Routledge.

Bers, M., Flannery, L., Kazakoff, E.R., y Sullivan, A. (2014). Computational thinking and tinkering: Exploration of an early childhood robotics curriculum. Computers \& Education, 72, 145-157. http://dx.doi.org/10.1016/j.compedu.2013.10.020

Bers, M., González, C., y Armas, U. (2019). Coding as a playground: Promoting positive learning experiences in childhood classrooms. Computers \& Education, 138, 130-145. https://doi.org/10.1016/j.compedu.2019.04.013

Bers, M. U., Seddighin, S., y Sullivan, A. (2013). Ready for robotics: Bringing together the $\mathrm{T}$ and $\mathrm{E}$ of STEM in early childhood teacher education. Journal of Technology and Teacher Education, 21(3), 355-377.

Brosterman, N. (1997). Inventing kindergarten. Henry N. Abrams.

Cejka, E., Rogers, C., y Portsmore, M. (2006). Kindergarten robotics: Using robotics to motivate math, science, and engineering literacy in elementary school. International Journal of Engineering Education, 22(4), 711-722.

Clements, D.H. y Sarama, J. (1997). Research on Logo: A decade of progress, 14(1-2), 9-46. https://doi.org/10.1300/J025v14n01_02

Cochran W.G. (1954). Some methods for streghning the common $\chi^{2}$ test. Biometrics, 10 , 417-451. https://doi.org/10.2307/3001616

Cohen, J. (1960). A coefficient of agreement for nominal scales. Educational and $\begin{array}{lll}\text { Psychological Measurement, } & \text { 37-46. }\end{array}$ https://doi.org/10.1177/001316446002000104

Cronbach, L.J., Gleser, G.C., Nanda, H., y Rajaratnam, N. (1972). The dependability of behavioral measurements: theory of generalizability for scores and profiles. Wiley.

Análisis observacional del desarrollo del pensamiento computacional en Educación Infantil-3 años mediante una propuesta de resolución de problemas con un robot de suelo de direccionalidad programada. Terroba, M., Ribera, J.M., Lapresa, D y Anguera, M.T. 
Denis, M. (2017). Space and spatial cognition: A multidisciplinary perspective. Routledge.

Diago, P. D., Arnau, D., y González-Calero, J. A. (2018). La resolución de problemas matemáticos en primeras edades escolares con Bee-bot. Matemáticas, Educación y Sociedad, 1(2), 36-50.

English, L.D. y Watters, J.J. (2005). Mathematical modelling in the early school years. Mathematics Education Research Journal, 16(3), 58-79. https://doi.org/10.1007/bf03217401

Fessakis, G., Gouli, E., y Mavroudi, E. (2013). Problem solving by 5-6 years old kindergarten children in a computer programming environment: A case study. $\begin{array}{llll}\text { Computers } \text { and } & \text { 8ducation, }\end{array}$ http://dx.doi.org/10.1016/j.compedu.2012.11.016

Gabin, B., Camerino, O., Anguera, M.T., y Castañer, M. (2012). Lince: Multiplatform sport analysis software. Procedia Social and Behavioral Sciences, 46, 4692-4694. https://doi.org/10.1016/j.sbspro.2012.06.320

Giaconi, V., Perdomo-Díaz, J., Cerda, G., y Saadati, F. (2018). Prácticas docentes, autoeficacia y valor en relación con la resolución de problemas de matemáticas: diseño y validación de un cuestionario. Enseñanza de las Ciencias, 36(3), 99-120. https://doi.org/10.5565/rev/ensciencias.2351

Hernández-Mendo, A., Blanco-Villaseñor, A., Pastrana, J.L., Morales-Sánchez, V. y Ramos-Pérez, F.J. (2016). SAGT: aplicación informática para análisis de generalizabilidad. Revista Iberoamericana de Psicología del Ejercicio y el Deporte, 11(1), 77-89.

Hernández-Mendo, A., López, J.A., Castellano, J., Morales-Sánchez, V., y Pastrana, J.L. (2012). Hoisan 1.2: Programa informático para uso en metodología observacional. Cuadernos de Psicología del Deporte, 12(1), 55-78.

Jiménez-Gestal, C., Berciano, A., y Salgado, M. (2019). Cómo trabajar la orientación espacial de modo significativo en Educación Infantil: implicaciones didácticas. Educación Matemática, 31(2), 61-74. http://doi.org/10.24844/EM3102.03

Kazakoff, E.R. y Bers, M.U. (2011). The impact of computer programming on sequencing ability in early childhood. En American Educational Research Association Conference (AERA), Louisiana: New Orleans.

Kazakoff, E.R., Sullivan, A., y Bers, M. (2013). The effect of a classroom-based intensive robotics and programming workshop on sequencing ability in early childhood. Early Childhood Education Journal, 41(4), 245-255. https://doi.org/10.1007/s10643-0120554-5.

Lapresa, D., Gutiérrez, I., Pérez-de-Albéniz, A., Merino, P., y Anguera, M.T. (2020). Interacción profesor-alumno-tarea en un programa de desarrollo de capacidades motrices en un adolescente con TEA: un estudio de observación sistemática. Journal for the Study of Education and Development. Infancia y Aprendizaje, 1-33. Online first, https://doi.org/10.1080/02103702.2020.1802148

Lee, K., Sullivan, A., y Bers, M. U. (2013). Collaboration by design: Using robotics to foster social interaction in kindergarten. Computers in the Schools, 30(3), 271-281. https://doi.org/10.1080/07380569.2013.805676

Lehrer, R., Giles, N.D., y Schauble, L. (2002). Children's work with data. En R. Lehrer $\&$ L. Schauble (Eds). Investigating real data in the classroom: Expanding children's understanding of math and science (pp. 1-26). Teachers College Press.

Análisis observacional del desarrollo del pensamiento computacional en Educación Infantil-3 años mediante una propuesta de resolución de problemas con un robot de suelo de direccionalidad programada. Terroba, M., Ribera, J.M., Lapresa, D y Anguera, M.T. 
Manches, A. y Plowman, L. (2017). Computing education in children's early years: A call for debate. British Journal of Educational Technology, 48(1), 191-201. https://doi.org/10.1111/bjet.12355

Mazas, B., Gil-Quílez, M.J., Martínez-Peña, B., Hervás, A., y Muñoz, A. (2018). Los niños de infantil piensan, actúan y hablan sobre el comportamiento del aire y del agua. Enseñanza de las Ciencias, 36(1), 163-180. https://doi.org/10.5565/rev/ensciencias. 2320

Pifarré, M. y Sanuy, J. (2002). La resolución de problemas entre iguales: incidencia de la mediación del ordenador en los procesos de interacción y en el aprendizaje. Journal for the Study of Education and Development. Infancia y Aprendizaje, 25(2), 209-225. http://doi.org/10.1174/021037002317417831

Pöntinen, S. y Räty-Záborszky, S. (2020). Pedagogical aspects to support students' evolving digital competence at school. European Early Childhood Education Research Journal, 28(2), 182-196. https://doi.org/10.1080/1350293X.2020.1735736

Resnick, M., Martin, F., Berg, R., Borovoy, R., Colella, V., Kramer, K., y Silverman, B. (1998). Digital Manipulatives: New Toys to Think With. Proceedings of CHI '98. ACM Press.

Rogers, C. y Portsmore, M. (2004). Bringing engineering to elementary school. Journal of STEM Education: innovations and research, 5(3).

Sackett, G.P. (1980). Lag Sequential Analysis as a data reduction technique in social interaction research. En D.B. Sawin, R.C. Hawkins, L.O. Walker, \& J.H. Penticuff (Eds.), Exceptional infant. Psychosocial risks in infant-environment transactions (pp. 300-340). Brunner/Mazel.

Sinclair, A. (2005). Las matemáticas y la imitación entre el año y los tres años de edad. Journal for the Study of Education and Development. Infancia y Aprendizaje, 28(4), 377-392. http://doi.org/10.1174/021037005774518983

Strawhacker, A. y Bers, M. (2015). "I want my robot to look for food": Comparing Kindergartner's programming comprehension using tangible, graphic, and hybrid user interfaces. International Journal of Technology and Design Education, 25(3), 293319. http://doi.org/10.1007/s10798-014-9287-7

Sullivan, A. y Bers, M. (2016). Robotics in the early childhood classroom: learning outcomes from an 8-week robotics curriculum in pre-kindergarten through second grade. International Journal of Technology and Design Education, 26(1), 3-20. http://doi.org/10.1007/s10798-015-9304-5

Terroba, M., Ribera, J.M., Lapresa, D., y Anguera, M.T. (2021). Education intervention using a ground robot with programmed directional controls: observational analysis of the development of computational thinking in early childhood education [Propuesta de intervención mediante un robot de suelo con mandos de direccionalidad programada: análisis observacional del desarrollo del pensamiento computacional en Educación Infantil]. Revista de Psicodidáctica, online first, 1-9. https://doi.org/10.1016/j.psicod.2021.03.001

Wing, J.M. (2006). Computational Thinking. Communications of the ACM, 49(3), 33-35. http://doi.org/10.1145/1118178.1118215

Wing, J.M. (2008). Computational thinking and thinking about computing. Philosophical Transactions of the Royal Society A: Mathematical, Physical and Engineering Sciences, 366(1881), 3717-3725. https://doi.org/10.1098/rsta.2008.0118

Zapata-Ros, M. (2015). Pensamiento computacional: Una nueva alfabetización digital. RED. Revista de Educación a Distancia, 46(4), 1-47. https://doi.org/10.6018/red/46/4

Análisis observacional del desarrollo del pensamiento computacional en Educación Infantil-3 años mediante una propuesta de resolución de problemas con un robot de suelo de direccionalidad programada. Terroba, M., Ribera, J.M., Lapresa, D y Anguera, M.T. 